\title{
Foresighting addiction and lifestyles in Europe 2030+
}

\author{
Jan Erik Karlsen • Antoni Gual • Peter Anderson
}

Received: 6 June 2013 / Accepted: 20 August 2013 / Published online: 6 October 2013

(C) The Author(s) 2013. This article is published with open access at Springerlink.com

\begin{abstract}
The paper links to the EU funded project titled «Addictions and lifestyles in contemporary Europe - reframing addictions project», acronym: ALICE RAP. The project invited 17 European and three international drug disorder experts to provide an initial vision and a reframing as to how scientific, technological and social advancement may impact on our understanding of addictions and lifestyles over the next 20 years («Vision 2030+»). The project design applied a combined approach using an in-situ substantial idea generation blended with well-established foresight tools. The data reported consists of qualitative narratives and quantitative assessments carried out by the experts in a 2 day electronic scenario workshop in Barcelona during May 2011. This research paper describes and analyses what these domain experts envisage as the premises, drivers, uncertainties and the most prominent images of this field in a prospective Europe. Four mini-scenarios were
\end{abstract}

Electronic supplementary material The online version of this article (doi:10.1007/s40309-013-0019-0) contains supplementary material, which is available to authorized users.

\section{J. E. Karlsen $(\bowtie)$}

University of Stavanger, 4068 Stavanger, Norway

e-mail: jan.e.karlsen@uis.no

\section{A. Gual}

Alcohol Unit, Psychiatry Department, Neurosciences Institute,

Hospital Clinic, IDIBAPS, Barcelona, Spain

e-mail: tgual@clinic.ub.es

\section{P. Anderson}

Institute of Health and Society, Newcastle University, Newcastle upon Tyne, England, UK

e-mail: Peter.Anderson@newcastle.ac.uk

\section{P. Anderson}

Faculty of Health, Medicine and Life Sciences, Maastricht

University, Maastricht, Netherlands

e-mail: peteranderson.mail@gmail.com designed to raise awareness that the future could go in different (possible, plausible, preferable) directions implying quite diverse individual and societal impacts. A main scenario describing images of the addiction and lifestyle scene in Europe 2030+ is offered, serving as a fourfold ideal type. By using a backcasting procedure the expert group launched an optimistic vision on the virtual future of European addictions and lifestyles, addressing distinctive collective values, long term planning and restitutive solutions for reframing the present drug disorder policy regime.

Keywords Foresight methodology $\cdot$ Future workshop · Expert assessments · Addiction and Lifestyles

\section{Introduction}

A major societal challenge

Contemplate addiction in Europe! This is what ALICE RAP ${ }^{1}$ aims to study and analyse. The development and place of a range of potentially addictive substances and behaviours represent major societal challenges to the cohesion, organisation and functioning of contemporary and future European society.

A specific ALICE RAP objective is to provide a stimulating vision and a global reframing as to how scientific and technological advancement may impact on our understanding of addiction and lifestyles and the measures to overcome

\footnotetext{
${ }^{1}$ The paper links to the EU funded large scale project titled «Addictions and lifestyles in contemporary Europe - reframing addictions project», acronym: ALICE RAP. The research project includes more than 140 researchers from 27 European countries, supported by a high-level group of international experts. It encompasses a wide range of coordinated quantitative and qualitative disciplines stretching across the humanities and social sciences and the biological and medical sciences, scheduled to be in operation from 2011 until 2016.
} 
related major societal obstacles in Europe over the next 20+ years. Our article addresses this foresighting objective.

Addictions, in general, are related to impulsive, unconscious behaviour [1]. As their numbers have increased over the last decades, they have become a focus of social, economic and political attention, sometimes polarising societies and politics. The motivations and interactions of addictions must be traced and analysed within social, cultural, family contexts and in individual characteristics and trajectories. How do social and living conditions, class trajectories, gender and different moments of the life course intervene? How do we develop policies of prevention and community intervention and policies for reducing risk and harm?

\section{Weak or strong signals?}

To deliver a sustainable policy response to addiction and lifestyles one has to assess signals on new developments. Obviously it is easier to pay attention to the strong rather than the weak signals emerging from the field of practice. In the perspective of the European Union (EU), the challenge originates from the impression that addictions are increasing in contemporary societies with subsequent concern at socio-economic and political levels. New forms of addictions are appearing, for example, Internet gaming. And, existing addictions appear to be increasing in size (for example gambling). The economic impact (lost productivity, and costs to health and criminal justice systems) [2], the political impact (for example, the debates around de-criminalization of illegal drugs) [3], and the societal impact (for examples, harms to others and the family, and stigmatization) [4] will need to build balanced policies that reduce the harm done by addictions and that enable both social integration and individual freedom.

To curb the adverse consequences of drug use and to cut drug-related crime, the EU drugs action plan (2009-12) addressed rather wide-ranging measures to strengthen European cooperation [5]. Concurrently with the large-scale ALICE RAP programme, the plan gave a strong signal on the need for change in Europe. Also the recent report from the high-level Global Commission on Drug Policy points in the same direction; the time for action is now [6]. However, the actual signals on the effective means for change are much weaker, and do not yet reach evidence based consensus.

So, how can we know that what we conceive as weak signals and early warnings really are significant, and will persist? We do not. However, we may apply the best of our knowledge and professional insight to discuss them. And subsequently, which conditions are more certain and which are more uncertain? We will need to concentrate on the segment of attention defined under the narrower heading of «early detection» (of changes to come), encompassing such typical and well documented methodologies as 'weak signal' analysis, 'early warning' assessment or 'early detection' of changes, and more recently, 'seeds of change' 77$]$ to envisage the future European addiction and lifestyle scene.

\section{Material and method}

\section{Domain expertise}

The material of this article consists of data reported by assessments carried out by 20 European and international addiction experts in a 2 day electronic workshop session, during May $2011 .^{2}$ The experts were addressing addiction policy, the drivers on lifestyles and addiction and the interplay between addiction policy and society at large. Hence, this article will report on images of the European addiction and lifestyles scene in $2030+$.

The experts engaged in the workshop were nominated on the basis of their merits in the domain of research on addiction and lifestyles. In this context it is not knowledge about the future per se which is the selection criterion. Rather, the experts were invited to participate based on their documented, updated and reputed insights on contemporary addiction and lifestyles in Europe. Unique, evidence based knowledge (i.e. domain expertise), rather than common, routinized knowledge was the criterion for selecting each expert [8]. What we hoped to elicit via the use of such an expert group was novel and pregnant images of the medium-long term future, conducive to a reframed understanding of the significance and impact of addiction and lifestyles on European society.

Not unexpected, the expert panel allotted much time to discuss the definition of the addiction concept per se. Does the WHO statement that addiction is [9],

Repeated use of a psychoactive substance or substances, to the extent that the user (referred to as an addict) is periodically or chronically intoxicated, shows a compulsion to take the preferred substance (or substances), has great difficulty in voluntarily ceasing or modifying substance use, and exhibits determination to obtain psychoactive substances by almost any means...

suffice when it comes to describing and understanding the full range of addictions, e.g. internet gaming and gambling, eating disorders, megarexia, etc.?

\footnotetext{
$\overline{2}$ The foresight workshop on addiction and lifestyles took place at the Science museum CosmoCaixa in Barcelona 26-27 May 2011, engaging 17 European and three international drug abuse and addiction experts. The experts were nominated amongst the 130 participants invited to the ALICE RAP kick off in Barcelona 23-26 May 2011, representing all six substantial areas of this project; i.e. counting addiction; determinants, governance, ownership and business of addiction, and addicting the young. See https://sites.google.com/site/alicerapproject/home.
} 
Addiction is not a diagnostic term in ICD- $10,{ }^{3}$ but continues to be very widely employed. It will be restored in DSM$\mathrm{V}$ [10], and maybe also in ICD-11. However, the expert panel commented that addiction presents itself as a primitive concept in scientific terms, i.e. it has low concept validity. Besides being neither a specific diagnosis nor a very strict concept for scientific purposes, it bears different connotations when applied by domain experts or by the layman. The experts engaged into broad discussions about the need for a more exact, measureable, comprehensive and consensus based concept applicable for the understanding of addiction in a multidisciplinary approach such as the ALICE RAP. When discussing a broad range of addictions (e.g. gaming and gambling, social media, physical exercise, sugar urges, eating disorders, etc.), sub-concepts and synonyms of addiction stemming both from the field of practice, the public debate and the sciences, like compulsion, craving, dependency, urge, devotion, dedication, sensation seeking, etc. were introduced. Likewise, the WHO definition does not entail the leisure part of addiction, i.e. addiction as a means of pleasure. For ALICE RAP, it is imperative to develop and apply a conception of the addiction phenomenon that also includes the motives for entering into an addictive mode of behaviour, not only focusing upon harms reduction. Perceptions of addictions, behavioural patterns and interaction contexts in which they occur, and even the experience of addiction itself, are affected by how the phenomenon is conceived $[11,12]$. This may in turn colour the concept and the perceptions of addiction and lifestyles as a social trend and assumedly also impact the policy strategies developed to cope with them. The experts took this inadequacy and ambiguity of the addiction concept into consideration when carrying out their discussions and subsequent foresight assignments.

\section{Electronic knowledge production}

The project design applied an electronically combined approach using an in-situ substantial idea generation blended with well-established foresight tools, called E-lab [8]. The ELab consists of a methodology database and a set of laptops in a local area network, supported by experienced facilitators. It is portable and can be set up anywhere. It allows for parallel input of data from all participants, anonymity, instant availability of input data, and structures the ideas in a stepwise manner. Participants can simultaneously generate and communicate ideas, comments, oppositions, etc. This eradicates waiting to take turns to «speak» and facilitates electronically storage of all input data. The technique is nominal in the sense

\footnotetext{
$\overline{3}$ International Classification of Diseases (ICD) was endorsed by the Forty-third World Health Assembly in May 1990 and came into use in WHO Member States as from 1994.
}

that there is little interpersonal or group interaction outside the electronic meeting itself and the expert group is composed for the exercise only.

This electronic workshop system has been designed to enhance group effectiveness, efficiency and satisfaction. It delivers increased interactivity and participation by parallelization, increased openness and less personal prejudice through anonymity, more sophisticated analysis by voting and analysis in real time, and by the end of the day automatic, comprehensive, neutral documentation retrievable from the computer [13, 14]. Contributions were directly entered by the participants and immediately visible on screen to everyone in anonymous format. By sidestepping social barriers (anonymity) and overcoming limitations of process (parallelization) more ideas were generated and shared with less conformity than in a traditional brainstorming or brainwriting session. The benefits of electronic brainstorming increase with group size [15].

The expert group session imbedded a series of knowledge elicitation techniques; creativity tools, decision tools, assessment instruments, consensus methods like Delphi techniques, expert group tools based on nominal group techniques, etc. All techniques were converted onto electronic templates concentrating on addiction and lifestyle elements. This approach also supported brainstorming, developing univocal terminology, categorising of ideas, and evaluation of these, using multiple criteria and techniques.

Discussion and the exchange of experiences are the core elements of a scenario workshop [16, 17]. The assignment for the addiction and lifestyle experts was to deliver images and narratives of the European future (i.e. a Scenario) and a «Vision 2030+». Initially, drivers that might have an impact on future addiction and lifestyles, for example economic adaptability, scientific progress, novel regulations, opinions and attitudes amongst people, etc. were listed in the initial brainstorming exercise. Next, all variables were classified either as factors (i.e. structural trends) or actors (i.e. recognizable and purpose-oriented players). While in the first phase emphasis was placed on creativity and the free exchange of ideas, the second step was to reduce the initial list of variables and indicators (actors/factors) to a more manageable set for further elaboration in the subsequent steps.

Equipped with the extensive list of drivers, actors and factors the experts were separated into four breakout groups, to construct brief (10 lines) narratives on future addiction and lifestyle images. First, the moderators explained how to apply a particular template to organize the writing of the narratives. Next, the experts should imagine they were situated in 2030+ and describe a few different synopsis-like «mini-scenarios» for the most significant aspects of their choice, including which actors who possibly had influenced on the trajectories and outcomes. After writing the mini-scenario narratives, the groups should classify them by means of probability and impact/importance on the template. 
For the relevant elements selected by the groups, alternative future trajectories were sketched, involving aspects such as increase in individualism and social segregation, social awareness, new and powerful players on the scene, the economic slump impacts' on research and development (R\&D), the use of information and communication technologies (ICT) changes, etc. Subsequently the number of variables was reduced again to 3-4 per mini-scenario. Decisive for this reduction were the dimensions uncertainty of and impact on the outcome, assessed by the experts in an anonymous voting session. A suitable and noteworthy name was chosen for every partial scenario by the breakout groups. They were described using a standardised template, the most prominent actors and factors were commented, together with a rough sketching of a plausible time line. The templates confined columns for time lines and for comments on envisioned European watersheds in the addiction and lifestyles trends unto 2030+. Finally, all mini-scenarios were presented and briefly discussed in a plenary session by the end of the first day.

The methodological clue of the E-lab assignments was to elicit experiences and ideas about the shaping of future addictive lifestyles by applying a mix of foresight methods and tools. At the end of the 2-day session, a report was automatically generated containing everything that was written during the workshop. In that way the group was not dependent on a secretary to pick out what might be the most essential elements of the meeting. This article is based on the recorded output from the experts' ideas, assessments and scoring.

\section{Results - framing the future}

Initially, the experts identified a series of drivers, actors and factors that could influence the European addiction and lifestyle scene during the time period 2011 to $2030+{ }^{4}$ In all, 141 such items were identified in the brainstorming session, many of them also actuating comments and/or comments to comments. During a succeeding voting session the experts classified and ranked the items according to their relevance to addiction and lifestyles at large. These aspects were scored and ranked on a scale from 1 to 10 according to their probability and possible consequences or impacts, and finally the added scores were calculated. On the basis of the added scores, the most prominent items were clustered and assumed relevant input into the writing of the mini-scenarios. Eleven

\footnotetext{
${ }^{4}$ Basis for the workshop was presumptions made for one generation ahead, thus reflecting the situation beyond year 2030+. This requires a set of actions to be taken shortly, subject to European policy, and to become effective around year 2015. Basically, societal conditions were considered, and only to a lesser extent were technological (e.g. ICT, medical technologies) aspects addressed.
}

such mini-scenarios were initially produced as output of the workshop (see Annex), out of which four cases conducive to the construction of a main scenario (c.f. Fig. 1) are elaborated in this article.

Mini-scenarios on drug addiction and lifestyles

Mini-scenarios (as qualitative narratives) are not designed to provide clear-cut forecasts of the addiction policy of Europe. What they are designed to do is to raise awareness that the future could go in very different directions and alert people to the potential impact and wider implications of a variety of trends across the board. The narratives allow users to ask questions, see connections and raise issues that might otherwise not get raised. They provide a context based on plausible outcomes, they are there to explore not predict those outcomes, and they aim to challenge current thinking and raise further questions. $^{5}$

Four mini-scenarios offering differing images on addiction and lifestyle in $2030+$ are now described. The first image deals with the highly plausible and consequential development related to advances in neurological biological sciences to curb the harmful effects of drug use. A different roadmap stems from the changes in values, attitudes and measures produced in a collective atmosphere based on the idea of equality in social order. A third image where addiction and lifestyles are piggy-backing on the envisaged ICT-based infusing of most social arenas and activities, is representing sort of an inbetween perspective connecting the two preceding miniscenarios. A fourth narrative deals with the impact of increasing social inequality. All narratives are presented here as raw, non-edited text material.

\section{MS1: Better drugs to deal with drug disorders}

The further development of neurological biological sciences is leading towards the discovery of more effective drugs to diminish drug related harm. We may expect that drugs are developed to deal not only with dependence and cravings but also to take away the negative effects of consumption.

The availability of these kinds of drugs will automatically lead to a more relaxed attitude in society towards drugs and users (fewer stigmas). In $2030+$, we would expect that addictions are treated in medical facilities with no discrimination.

Within each of the four subgroups significant drivers, and actors and factors were scored. On this basis, the experts described a first mini-scenario (c.f. text box MS1), branded

\footnotetext{
${ }^{5}$ When reading the (mini-)scenarios, it is important to remember that they are written as though we are in the future, we should think of them as stories that might appear in the science section of a newspaper review of the year. All of them are set 20 years into the future, i.e. $2030+$.
} 
«Better drugs to deal with drug disorders», having a high probability (8) and a high impact (9).

This image assumed three drivers to be significant;

a) The development of effective pharmacotherapies. Those which are effective are most likely to be essentially maintenance, e.g. another stimulant instead of amphetamine, so they become a way of 'taming' rather than reducing or eliminating drug use.

b) The routine use of pharmacotherapies in medical care, also recent recognition of their limitations and recognition that psychosocial interventions have important role, even if their effect is rather generic.

c) Advances in biological science (e.g. discovering additional neurological processes and factors related to addictions) and pharmacology (e.g. new drugs, more effective drugs dealing with harmful effects). Such neurological advances give better understanding of the biological mechanisms involved which can lead to better treatment but also more effective prevention.

The experts identified neuroscientists to be the more influential actors shaping such a future, supported by funding agencies backing appropriate research, the pharmaceutical industries funding and marketing the results from this research and the clinicians learning and implementing new treatments. In a way this may almost look like a zero-addiction vision of Europe in 2030+. However, this image needs a strong support from science and industry to materialize, the experts claimed.

The second mini-scenario assumed a series of drivers related to new information technology; methods of sale, access to rapid information about good and bad drugs, health information, cessation services group support and ways of enhancing behavioural interventions monitoring and building new skills, use of social networking for public education and use of e-technologies for prevention and treatment. The expert group rated their image (c.f. text box MS2), named «Addictions in the e-age» to have a high probability (8), however having a medium impact (6) on future. In the eyes of the experts, several actors are assumed to impact on this future. The industry, both the IT and the drug industry profit from continuous development of new products and through e-commerce, as well as do the health providers by means of e-health tools. Also, mass media actors impact on the development; content providers adjust to new types of telling narratives, new demand of knowledge and knowledge distribution.

\section{MS2: Addictions in the e-age}

The consumer of the next 20 years will increasingly address the Internet to buy psychoactive drugs; game online and once addicted will address the opportunity to become less dependent through web site, e/ intervention and telemedicine.
Ready and immediate access to pleasure fulfilment, leisure and information. Information technology impacts on market; people perceive spatial, temporal dimensions and their own body. New forums of simulation, virtual environments with new stimulus. New attitudes towards sensations. Identity, body and self. The virtual life is addictive in itself; repetitive actions in IT. New types of realities. Challenge: balancing, individualisation, social isolation, but also new opportunities to socialize. Loss of human physicality (smell, touch, pain). Living in present, inability to immediacy: loss of contact with the past and the future, discounting the future. Challenges notion of Europe, of national states? Use of technology to take care of elderly population. Reducing the burden on drug treatment services by using robots.

All this facilitates new direct democracy, liberty of access, voice heard, and possibility to publish widely by a single «normal» person. Opportunity to make messages available and easy to understand, but also more «dumber»? Simplification? Gathering intelligence, gathering people's opinions -> customise content to the needs of demands and desires.

Today's ICT tools' development prolongs and improves; touch screen, Twitter, Facebook, apps, digitalized techniques in movies and TV, PC gets smaller, etc. In 2015, we will have WiFi everywhere, $80 \%$ of Europeans will have small PC devises with touch screen. 2020 will experience advances in biotechnology, level of consumption can be checked and monitored, and more screening and testing will be performed. In 2025 , the experts envisage that messages will be submitted by images and voices instead of writing. The situation in $2030+$ reveals a collective shock; huge amounts of brain tumours because of mobile phones and microwave ovens are discovered, drastically curbing the confidence in the e-society's technology platform.

\section{MS3: Alice in an equal wonderland}

Equal access to screening, early intervention and treatment are common. Better general health and education and leisure in the population. Negative outcomes declining, more democratic representation, more political engagement by the public, people feel the world is fair, that they have opportunities. They are making better choices around health and public health. Drug use is legalised, state regulation of quality of substances and prices. And regulated harm potential.

Population has found healthier ways of finding a «high» (they have more access and are healthier and intellectually stimulated).

Youth will always test limits (but there is a potential to test limits at an intellectual level). There will be more pharmacological solutions to counter-act the harmful aspects of drugs. There is universal access to mobile information technologies, which provide more access to health information and interventions. And individualised interventions that will reduce susceptibility to effects of drugs (e.g. breathalyzer connected to car bicycle, depending DNA). Those who do not fit in (e.g., due to a limitation, a rare disorder) might be more stigmatised, but buy in to harm reduction could potentially help.

The third example envisages the future as opposite to or at least quite different from today's society, labelling the miniscenario «Alice in an equal wonderland». 
This narrative is paraphrasing both the ALICE RAP acronym and the concept of an ideal, addiction regulated future, treating every addicted person on an equal footing. The experts deemed this image with a low probability (4); however having a great impact and importance (8) if realised. The public is seen as the most significant actor, insisting on fairness and democratic ways, demanding more from themselves and their community, state and law being more accountable, regulation and laws promoting equality, and industry being more responsible.

The expert group described forerunners of such an image to be three current events; the UK coalition government, the «Arab spring» and the decriminalization of drug use in Portugal, all three signals of a new turn in attitudes towards addiction and social equality. In 2015, the experts postulate that the Portugal experiment produces data and is replicated in other countries, and a Jasmine revolution will take place. ${ }^{6}$ In 2020, an emerging technology revolution is making available free health information and interventions.

This image is not presupposing any step change in pharmacological measures and solutions, just a gradual improvement in medical technology to deal with harmful aspects of addiction. However, there is a change in attitudes towards more collective responses, including a more responsive public health, a fading drug trend and an inclination towards new forms of sensation seeking, a liberalisation of drug use, and a far better access to appropriate ICT-based health information. It is interesting to notice that the experts deem the significant factors driving the addiction phenomenon to be on the societal level.

\section{MS4: Impact of increasing social inequality}

Welfare state is weakened and a resultant increase in inequality within societies. Increased stigmatisation of drug use among the poor, although not necessarily increased use. Unequal access to social and institutional supports.

Demographical developments, increasing disparities between rich and poor. Marginalisation, dislocation, weakening of the welfare state, selfishness and competitions, crises of family.

Role of the state: To protect the wealthy world instead of caring for the weakest members. Higher criminality. Ageing population in combination with less care. Drugs - it's your own fault. Sedation of the lower classes. Ghettos with addicted inhabitants. The importance of «the othen» for making «us» stronger. Free market economics. Individualism. Consumerism. Areas in Europe: All will go towards more market. Loss of religion, ideology, moral values. Climate change, global epidemics. Polarization: Unstable situation, more criminality, more drugs, more addictive behaviours, a counter movement.

\footnotetext{
${ }^{6}$ Princess Jasmine is a fictional character of the 1992 Disney film 'Aladdin'. She is a very spirited young woman with a mind of her own and yearns for freedom just like most teenage girls. The Jasmine flower has many symbolic connotations and applications, mostly reminding us about the beauty of life besides producing pleasant odours that scent our ambience.
}

The fourth mini-scenario launches the current economic crisis of Western Europe as a hinterland to the future state. Will the crisis lead to less or more drug use?

The outcome may depend on which driver will cause the strongest effect; reduced income or the psychological stress induced. It relates to incremental, but significant socio-economic and demographic changes. The welfare state is replaced by a market driven society where more responsibility is put on the individual and his resources. Inequalities increase: the rich get richer, the poor get poorer; there are deeper gaps among social classes. This image is deemed having high (9) impact and importance and medium (6) probability. It describes a development very much on the opposite scale as MS3 above. The most significant actors who possibly could impact on this future state are bottom-up progressive social movements, direct acting for the support of the welfare state and advocating for the poor. Also right wing populist movements and their supporting powerful economic actors could impact on the social inequality aspects by reinforcing certain neo-liberal trends. However, the economic crisis speeds up the shrinking of public resources, leaving more to the responsibility of the individual.

The eleven mini-scenarios, ranked on the basis of estimated probability and impact were plotted in a matrix to assess possible overlaps and similarities. From all mini-scenarios, including the four illustrated above, the assembled expert group started to cultivate a main scenario. Input was the long list of drivers, the assessed uncertainties and impacts, and the naming and actions of significant actors who could impact on the future state. Besides, the mini-scenarios could preferably serve as narratives for the consecutive main scenario.

\section{Pulling the strands together: the main scenario}

As a concluding assignment, the experts were summoned and asked to construct a main scenario about the situation in $2030+$ and indicate elements on the road from today towards that year. In doing so they were asked to include relevant stakeholders and players as part of the descriptions. When appropriate, the experts were asked to combine mini-scenarios to construct the different main images. Likewise, a main scenario needed storylines, i.e. narratives that presented the important aspects of the images, including the relationship between driving forces and events of the scenario. Hence, the scenario should encompass ideas of the alternative futures in terms of political environment, public acceptance, markets, values, social conditions, etc.

Prior to the plenary discussion on the second day, the moderator team had run a preliminary analysis of the 
input produced by the experts during the first day. The narratives from the mini-scenarios together with the identified drivers, timelines and significant players were used as input to elaborate a more full-grown scenario and storylines. The moderator introduced the group to the image construction phase using a two axes scenario technique, presenting four (uncertain) futures as an illustration. This didactical scenario presumed opposing views on values underlying the perception of addiction (as deviance or sickness) and on the sanctions (as repressive or restitutive) executed by the society. Equipped with this kind of reasoning and the two axes technique, the expert group discussed and gradually developed ideas which were summoned into a situational scenario $[18,19]$, describing the addiction and lifestyle scenes of a 2030+ Europe.

From the identified drivers, uncertainties, actors and the subsequent mini-scenarios the experts developed images of the addiction and lifestyle scene in Europe 2030+. By means of the two axes method of scenario writing, a matrix was built up [20], depicting plausible images of the future. Preferably, the factors chosen for the axes should be 'high uncertainty/ high impact' to ensure that the four images defined by their intersection are clearly differentiated. In turn, these images were to be elaborated into scenario narratives, reflecting the influence of other events and trends beyond and in addition to those depicted on the two axes.

The two most prominent drivers of change were assumed, firstly to be the decisive values of European citizens; will there be a priority to self or to community and secondly, the nature of response from society, will there be a dominance of reactive, short-term responses or a forethought, long-term systemic change? This approach generated four contrasting scenarios relevant to our field of interest by placing a major factor influencing the future on each of the two axes, which cross to form four quadrants (see Fig. 1).

\section{Nature of response}

\begin{tabular}{|c|c|c|}
\hline & React and mitigate & Anticipate and prepare \\
\hline & Inequality prevails & Vocal player's arena \\
\hline $\begin{array}{l}\text { Individual res- } \\
\text { ponsibility first }\end{array}$ & $\begin{array}{l}\text { MS2 } \\
\text { Intense individualism and } \\
\text { short term reactions to } \\
\text { addiction }\end{array}$ & $\begin{array}{c}\text { MS4 } \\
\text { Market driven society, } \\
\text { prepares for future } \\
\text { challenges of addiction }\end{array}$ \\
\hline Values & $\begin{array}{l}\text { Ad hoc treatment } \\
\text { society } \\
\text { MS1 }\end{array}$ & $\begin{array}{c}\text { Solidarity prevails } \\
\text { MS3 }\end{array}$ \\
\hline $\begin{array}{l}\text { Social res- } \\
\text { ponsibility first }\end{array}$ & $\begin{array}{l}\text { Inclusive debate, challenges } \\
\text { of addiction met when they } \\
\text { occur }\end{array}$ & $\begin{array}{l}\text { Feelings of societal } \\
\text { vulnerability engender large } \\
\text { scale, long term actions on } \\
\text { addiction }\end{array}$ \\
\hline
\end{tabular}

Fig. 1 The Scenario Axes of Addiction and Lifestyles in Europe
Of course, the very concept of a future lying $20+$ years ahead calls for a discussion about uncertainty, ambiguity and complexity. A four-fold world, as depicted in Fig. 1 will not be loaded with details on every of these dimensions. Rather, it is a simplification of futures, an ideal type in the language of the classical Weberian reasoning [21, 22], being a methodological and conceptual device that can be understood as a logical summary or recapitulation of several trends and aspects of social life. Therefore, our four scenarios as ideal types are neither a normative description of a preferred state nor a description of how the world actually will look like.

When intersecting 'individual responsibility first' with 'react and mitigate' the first scenario, named «Inequality prevails» emerges as depicted in the upper left quadrant. It images a future state with intense individualism and short term reactions to addictions. Competitive, innovative actors dominated by short term reactions to change, create a society in which health and lifestyle inequalities become very apparent. This scenario overlaps with; although it is not totally equivalent to the narrative described in MS2 about the development of addiction in the e-age. Individualisation, social isolation, and living in the present characterise this image.

«Vocal players' arena» combines the individual responsibility with a longer anticipative time perspective and preparation, as in the upper right quadrant. Outspoken and significant actors have set priorities in an individualistic, market-driven society, which invests in long-term planning, preparing for future challenges of addiction and change in lifestyles. MS4 describes in bullet terms much of the same development; it is a society in which the fittest survive.

Anticipated value profiles may also be conceived as putting social responsibility first. On the shorter term axis intersecting the immediate reaction and mitigation, we find the «Ad hoc treatment society», (see lower left quadrant). This societal formation encourages an inclusive debate in which challenges of addiction and unforeseen lifestyles are met by working together in therapeutic and/or treatment communities. However, challenges of addiction are met only when they occur and when they cause social unrest or health policy disturbances. MS1 describing better drugs, encompasses much of the same perspective. It is an image based on technology optimism; eventually new advancement of medicine will negate the harmful effects of today's drug use.

The last scenario (see lower right quadrant) entails a situation where reactions against individualism create a growing sense of responsibility to the community, conducive to drug users and addicted people. We name this scenario «Solidarity prevails». A growing awareness of irreversible societal vulnerability brings about long term and large scale planning for the future. Societal resilience calls for novel health policy, including preventive and intervention measures that accounts for the externalities of addiction. Much of this future optimism and hope is found in MS3. 


\section{Discussion}

\section{Forerunners of the future}

We often say that foresights are about thinking, debating and shaping the future $[23,24]$. We requested the experts to engage mostly into the thinking and debating stages of foresighting. It is apparently easier to think and debate the future than to shape it, but does this imply that our anticipation of how to shape the future contains more uncertainty the longer the time perspective? Arguably, the nearer the future, the easier it will be to shape it, so the action space is wider a month from now than it is perceived to be in $2030+$. At least, that could serve as a hypothesis.

In line with the reasoning of the forthcoming ALICE RAP project the experts brought some significant viewpoints to the discussion arena, including aspects of planning the future. Both the «Inequality prevails» and the "Vocal players' arena» scenarios focused on several factors that assumedly will influence addiction policy: cultural inequalities, liberalism and marginalisation. Cultural inequalities are seen as a source to conflict between different perceptions of acceptable drug use. The drug policy of the future will accordingly be flavoured by opposing interests between moral, fundamentalists' attitudes to drug use and attitudes concerning the right of the individual to enjoy drugs at «anytime and anywhere». In a historic perspective one may find that both such attitudes have influenced former drug policy since the policy itself has been shaped in the intersecting point between what drugs a society will accept; the costs for society on the one hand and the respect for the individual on the other.

In a multi-cultural society where different cultures affect the drug policy (as described in both these scenarios mentioned), Room et al. [25] claims ethnicity to be partly assigned and partly constructed and that drinking, use of drugs or abstention are ethnic markers. The ethnic identity is partly formulated by others, partly accepted and constructed by the one who complies with the identity. It may also appear important to demonstrate distance to other ethnicity. The conflicting and opposing interests described in MS4 (on social inequality) may also be interpreted as colliding opinions on moral, as well as expressing ethnic markers. Room et al. accentuate that status and power within ethnic groups both affects the drug use behaviour and the perception of what may be seen as a problematic use. The ideological basis influencing addiction policy is in MS2 and MS4 charged with incompatibilities. By using Room's argument about drugs as ethnic markers, the storyline of these mini-scenarios expressing inequalities and conflict on the perception of morality and a new Puritanism and neo-liberalism, may lead to a drug policy where larger portions of the population will not share the values expressed and where the legitimacy of the drug and addiction policy will be challenged.
MS4 inclines that the single individual has a right to indulgence or immoderation at anytime and anywhere, indicating a liberal attitude. The respect for the individual confronts the claim of the majority about individual freedom to enjoy, even enjoying drugs. The majority represents the ideological underpinnings of the ethnic proponents and the corresponding drug policy must therefore gain legitimacy by the majority. Arguably, such an addiction policy might challenge the opposing value basis of the minority. The dilemma between considering individual autonomy and freedom for the few versus the many, may be seen in line with what Sulkunen et al. [26] describes as the dilemma in preventive action; «The dilemma in problem prevention - the promotion of the public good versus market and individual freedom - is accentuated by increasing costs due to improved medical capacity to treat health problems». The authors claim that social development in Western Europe has embarked on a common project of modernisation where social ties have kept the moral society together, but this strategy is now ended. Inside the welfare state new public management (NPM) shall replace old fashion bureaucracy [26]:

The role of public institutions in this new strategy is not bureaucratic control but the empowering of citizens and market actors in order to prevent problems. Its reverse side is the criminology of the other, which means limiting the citizenship rights of particular groups, dehumanizing deviants and excluding them from normal society with long prison sentences, and maximizing the visibility of penal consequences.

Such a drug policy can be characterised as «moral management of self» with «the moral management of others» as the flip side of the coin. Sulkunen et al. [26] claim that the rhetoric of NPM demonstrates the challenges of a drug policy addressing sensitive themes and phrases this as the «ethic of not taking stand» and warn against such a development. Emphasis is placed on the individual and its mastery and self-control. Arguably, such drug and addiction policy will not reflect societal conditions that may improve the situation for people in the risk zone for problematic alcohol consumption or drug use. A drug policy built on «the moral management of others» may result in exclusion and marginalization of those carrying the problems. Such a trajectory is expressed in the MS4, «Impact of increasing inequality», where «us» - the survivors have all the credentials and may achieve the project of «moral management of self». Although the society is perceived as more complex than before the drug and addiction policy will not focus on changes at the society level, but on continued pitying those who cannot make it through «the importance of the 'others' for making 'us' stronger». 
The scenario concerning how the addiction policy may be influenced by the vocal elite represents a new development (se Fig. 1, upper right quadrant). A present argument against the addiction policy is that it has been popular, but not effective. Measures documented effective have not been deemed implementable. When it comes to alcohol [25]:

The crucial need, from a public health perspective, is for a regular means of coordination whereby prevention of alcohol-related problems is taken fully into account in policy decisions about alcohol controls and other regulation of the market for alcoholic beverages.

Still, this research is delimited to alcohol and there is a need for more studies [27], a recommendation the ALICE RAP expert group supported fully.

Varying perceptions and public understanding of addiction may have great influence on the handling of the challenges and harms connected to the phenomenon, ranging from penal or therapeutic measures to individual or societal strategies, as well as impacting on the time perspective for action. This may have consequences for our understanding of addiction as interplay of social and biological processes and outcomes. Hellmann $[28,29]$ demonstrates that media coverage on addiction has introduced a new dominant view on addiction as a problem for the individual rather than for a marginalized group or for the society at large. This view is also reflected in our MS2 and MS4, as well as in the scenario called «Inequality prevails» from Fig. 1. Arguably, reaching a common denominator for the concept and novel phenomenon of addiction is a prerequisite for a future oriented public health policy.

\section{Conclusion}

Thinking and debating the future

It is fair to say that the ALICE RAP expert workshop focused on the developing stories of possible futures, as well as on probable, plausible or preferable futures of the European addictions and lifestyles scene. Further, the expert process stimulated mind boggling and imaginative scenarios, conscientiously applying wild cards and other creative techniques. The workshop was also explicit in its premise, that the result of the scenario building process did not end up in some academic cul-de sac, but should be an input to shape the future. Arguably, the experts attempted to meet the comprehensive challenges of foresights, particularly the thinking and debating aspects of the future of the addiction field.

The expert group engaged into some sort of retrospective thinking (from today back to the 1990s) and backcasting (from 2030+ back till today) when discussing elements of a vision for the $2030+$. Foresighting as construction of the past means a sort of backcasting while at the same time applying a perspectivistic approach [30]. The backcasting is an elaboration of the virtual history, stepwise identifying the optimal pathway from $2030+$ to the current baseline [31-33]. In the conclusion of the workshop the experts engaged in integrating its best hindsight and foresight in aligned action, the challenge was to extract a «Vision $2030+»$, possibly embedded in the prior thinking and debating of future addiction and lifestyles. The goal was not to find the majority opinion, but to arrive at a vision that reflected the thinking of the diverse experts participating. Not surprisingly, the «Alice in equal wonderland» mini-scenario attracted most attention as did the «Solidarity prevails» situational scenario. Their focus on collective values, long term planning and restitutive solutions paved the way for a «Reframing of the European addiction policy». Of course, this is a normative statement, but with a $20+$ year horizon, visioning may in the opinion of the experts produce European policy measures conducive to reframing the challenges of addiction.

The benefit of the expert views on futures drug policy may be enhanced when analysing the mini-scenarios in relation to similar recent phenomena or development trajectories. The perspectives offered by Room et al. [25, 27] about drug use as a ethnic marker in multi-cultural societies and what consequence NPM has for drug policy are conducive to our analysis. Also assessing consequences of the ethic of not taking stand, proposed by Sulkunen et al. [26] contribute to prevent an unwanted development. Even though the welfare state models of the USA, Western Europe and the Nordic countries are different, Lee et al. claim that the health policy at the macro level must contain effective health promotion addressing problems that stem from behaviour-environment interaction, rather than to «...enrich those who profit from the War on Drugs and the unchecked expansion of the penal system» [34]. Instead of «Looking Back and Moving Forward» which is the contribution of Lee et al. to the American drug policy, we will on the basis of the visions of the European expert group, argue that the coming next 20 years will welcome an expert based drug and addiction policy by looking both back and forward when moving towards the futures.

Although belonging to various scientific fields, the experts landed on a societal rather than an individual perspective when finalising the main scenario and the vision statement. Recognising that much (but by far not all) of recent research on addiction and lifestyles is centred on theories in medicine and psychology [1], the positioning of this expert team is both challenging and interesting when it comes to future European policy and measures to curb addiction. Both the need for a reframing of policy and a conceivable paradigm shift in the understanding of addiction [35] are implications touched upon during this electronic expert assessment. However, to address that in full is another story. 
Declaration of interest: Antoni Gual has received honoraria and travel grants from Lundbeck A/S, Janssen-Cilag and D\&A Pharma. Peter Anderson and Jan Erik Karlsen have no declarations of interest to state.

Open Access This article is distributed under the terms of the Creative Commons Attribution License which permits any use, distribution, and reproduction in any medium, provided the original author(s) and the source are credited.

\section{References}

1. West R (2006) Theory of addiction. Blackwell Publishing \& Addiction Press, Oxford, pp 9-28

2. Laslett A-M, Catalano P, Chikritzhs Y, Dale C, Doran C, Ferris J, et al (2010) The range and magnitude of alcohol's harm to others. Fitzroy, Victoria: AER Centre for Alcohol Policy Research, Turning Point Alcohol and Drug Centre, Eastern Health

3. Olesen J, Gustavsson A, Svensson M, Wittchen HU, Jönsson B (2012) CDBE2010 study group; European brain council. The economic cost of brain disorders in Europe. Eur J Neurol 19(1):155-162. doi:10.1111/j.1468-1331.2011.03590.x

4. Roberts BR, Chen Y (2013) Drugs, violence, and the state. Annu Rev Sociol 39:105-125. doi:10.1146/annurev-soc-071312-145554

5. EC (2008) EU Drugs Action Plan for 2009-2012. European Commission, Brussels

6. War on drugs. (2012) Global Commission on Drug Policy, New York. www.globalcommissionondrugs.org. Accessed 20 February 2012. Archived by WebCite ${ }^{\circledR}$ at http://www.webcitation.org/65axBoOjy

7. Rossel P (2010) Making anticipatory systems more robust. Foresight 12(3):73-85

8. Karlsen JE, Karlsen H (2007) Expert groups as production units for shared knowledge in energy foresights. Foresight 9(1):37-49

9. Lexicon of alcohol and drug terms (2012) World Health Organization, Geneve. http://www.who.int/substance_abuse/terminology/ who lexicon/en/. Accessed 20 February 2012. Archived by WebCite ${ }^{\circledR}$ at http://www.webcitation.org/65axhG0gR

10. O'Brien C (2010) Addiction and dependence in DSM-V. Addiction $106: 866-867$

11. Becker HS (1953) Becoming a marijuana user. AJS 59(3):235-242

12. Elster J (1999) Emotion and Addiction: Neurobiology, Culture and Choice. In: Elster J (ed) Addiction, entries and exits. Russel Sage, New York

13. Nunamaker J, Dennis AR, Valacich JS, Vogel DR, George JF (1991) Electronic meeting systems to support group work. Commun ACM 34(7):40-61

14. Gallupe RB, Dennis AR, Cooper WH, Valacich JS, Bastianutti LM, Nunamaker JF (1992) Electronic brainstorming and group size. Acad Manag J 35(2):350-369
15. Dennis AR, Valacich JS (1993) Computer brainstorms: more heads are better than one. J Appl Psychol 78(4):531-537

16. Popper R (2008) Foresight Methodology. In: Georghiou L, Harper JC, Keenan M, Miles I, Popper R (eds) The handbook of technology foresight. Edward Elgar, Cheltenham, p 60

17. Krawczyk E, Slaughter R (2010) New generations of futures methods. Futures 42:75-82

18. Schwartz P (1991) The art of the long view: planning for the future in an uncertain world. Currency Doubleday, New York

19. Van der Heijden K (1996) Scenarios: the art of strategic conversation. Wiley, UK

20. Klooster SA V't, van Asselt MBA (2006) Practising the scenario-axes technique. Futures 38:15-30

21. Weber M (1946) From Max Weber: Essays in Sociology. Transl. and eds. H. H. Gerth and C. Wright Mills. Oxford University Press, New York, p 59. Reprinted by Routledge \& Kegan Paul, London,1970

22. Giddens A (2009) Sociology, 6th edn. Polity Press, Cambridge

23. European Commission (2002) Thinking, Debating and Shaping the Future: Foresight for Europe. Final Report Prepared by a High Level Expert Group. Directorate-General for Research, Brussels

24. Karlsen JE, Øverland EF (2010) Carpe futurum. Cappelen Academic Publishing House, Oslo

25. Room R, Babor T, Rehm J (2005) Alcohol and public health. Lancet 365(9458):519-530

26. Sulkunen P, Rantala K, Määttä M (2004) The ethics of not taking a stand: dilemmas of drug and alcohol prevention in a consumer society - a case study. Int J Drug Policy 15:427-434

27. Room R, Graham K, Rehm J, Jernigan D, Monteiro M (2003) Drinking and its burden in a global perspective: policy considerations and options. Eur Addict Res 9(4):165-175. doi:10.1159/000072223

28. Hellman M (2009a) From myth of marginality to portrayals of an addictive society: Reporting on addiction in the Finnish press (19682006). Addict Research and Theory: 1476-1492. Available from: Informaworld (28 January 2009)

29. Hellman M (2009) Designation practices and perceptions of addictiona diachronic analysis of Finnish press material from 1986-2006. NAD 26:355-372

30. Neumann I, Øverland EF (2004) International relations and policy planning: the method of perspectivist scenario building. Int Stud Perspect 5:258-277

31. Dreborg K (1996) The Essence of backcasting. Futures 28(9):813-828

32. Höjer M, Mattsson L (2000) Determinism and backcasting in future studies. Futures 32(7):613-634

33. Karlsen JE, Øverland EF (2012) Promoting diversity in long term policy development: the SMARTT case of Norway. Journal of Futures Studies 16(3):63-78

34. Lee PR, Lee DR, Lee P, Arch M (2010) U.S. drug and alcohol policy, looking back and moving forward. J Psychoactive Drugs 42(2):99-114

35. Alexander BE (2012) Addiction: the urgent need for a paradigm shift. Substance Use \& Misuse 47:1475-1482 